\title{
Acne scar management
}

\section{Dr. Ajith Prasanna Kannangara \\ Consultant Dermatologist, Base Hospital, Balapitiya, Sri Lanka}

\section{Introduction}

Acne is a very common skin disorder in adolescence and young adults. Mild and adequately treated acne often resolve without any complications, but chronic, severe inappropriately managed acne, results in permanent scaring. In fact post acne scarring is one of the main causes of facial scarring, leading to severe psychological distress. Unfortunately, scarring occurs early and may affect $95 \%$ of patients depending on severity of acne and delay in appropriate treatment. Understanding the basic pathogenesis of acne and the various types of acne scars is essential to optimize the management of acne scars. Since there is no single ideal procedure, various techniques are often used in combination to achieve the best outcome.

\section{Types of acne scars}

Post acne scars are polymorphic and various types of scars usually coexist in the same individual. It is very important that each scar be assessed individually and treated accordingly. The method of the intervention depends on the types of the scars, overall appearance of the face and budgets of the patient

\section{Depressed scar}

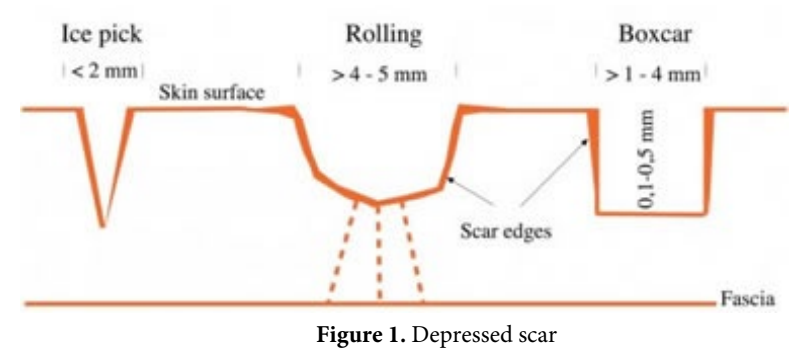

Icepick: Ice pick scars are narrow (less than $2 \mathrm{~mm}$ ), deep sharply marinated epithelial tracts that extend vertically to the deep dermis or subcutaneous tissue. The skin looks as if it has been pierced by an ice pick or sharp instrument. Ice picks scars seem to make a small, deep, "hole" in the skin. They are the most common acne scars that occur on the cheeks and have a "punctate" appearance. Ice pick scars develop after an infection from a cyst or other deep inflamed skin tissue and its way to the surface leaving a long column-like scar (Figure 2).

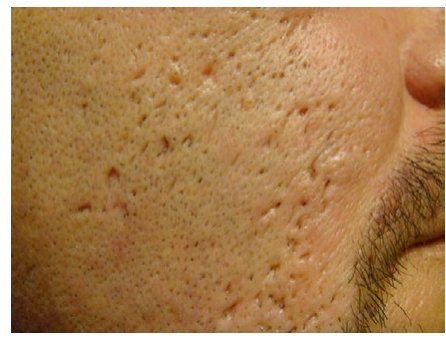

Figure 2. Icepick scar
Rolling: These are distensible, depressed scars with gentle sloping edges. Roling scar are shallow depressions that are best appreciated with a change in surface lighting. They can vary in size and often coalesce with neighboring rolling scars. Rolling scars are shallow, wide (more than 4 to $5 \mathrm{~mm}$ ) and have a subdermal fibrous tether. These scars are caused by damage under the surface of the skin. They give the skin a wave-like appearance (Figure 3).

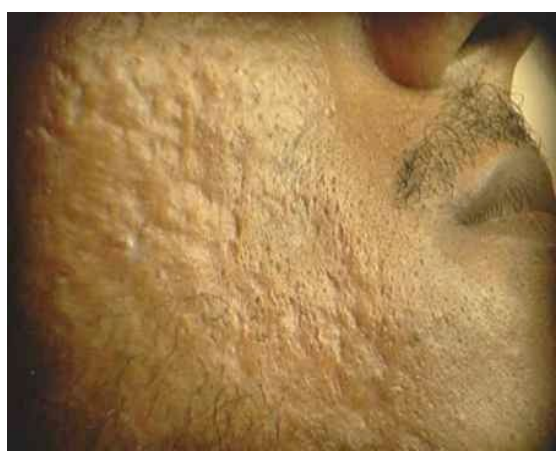

Figure 3. Rolling scar

Boxcar: Boxcar scars are angular scars with sharp vertical edges, and resemble the scars left by chicken pox. They may be shallow or deep, punched out, wide at the surface and at the base are most often found on the cheeks and temples (Figure 4).

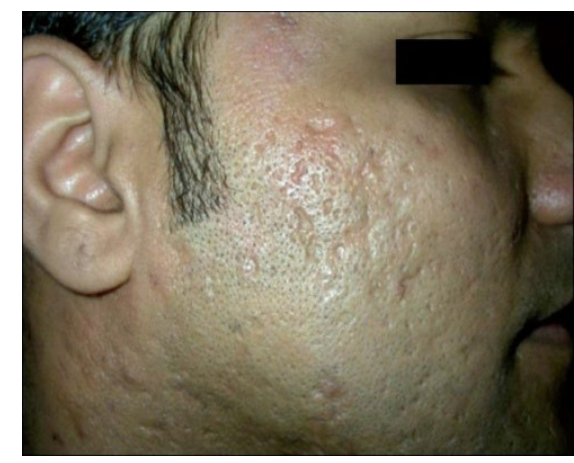

Figure 4. Boxcar scar

\section{Elevated scar}

Hypertrophic: These are elevated, fibrotic scars, more common in males and frequently seen in the mandibular area of the face and

Correspondence to: Dr. Ajith Prasanna Kannangara, M.D, Consultant Dermatologist andSpecialist in Anti-aging and Cosmetic Medicine Base Hospital Elpitiya, Southern Province, Sri Lanka; E-mail: ajithpkannangara@yahoo.com

Received: August 30, 2015; Accepted: September 24, 2015; Published: September 26, 2015 
back of the chest. Often the result of severe acne (cysts or nodules), they generally stay within the boundary of the original wound, and may decrease in size over time (Figure 5).

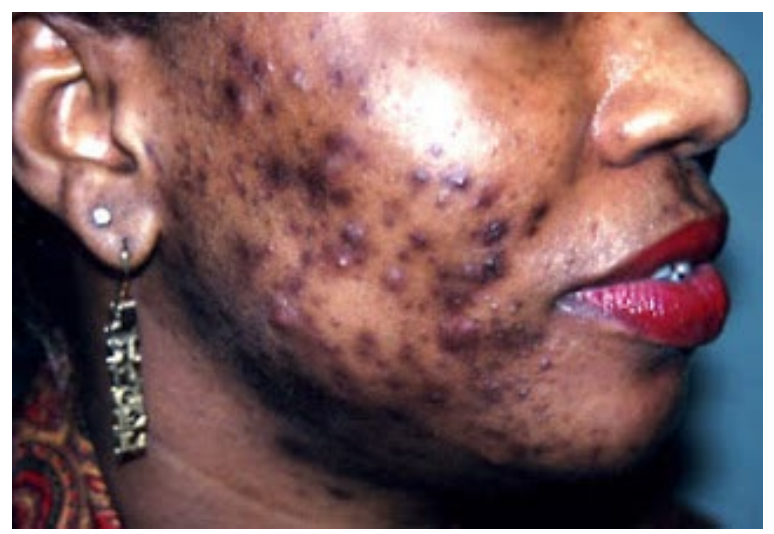

Figure 5. Hypertrophic scar

Keloidal: These keloids developing in acne lesions. They are seen more often in acne lesions. They are seen more often in males, on the back and chest (Figure 6).

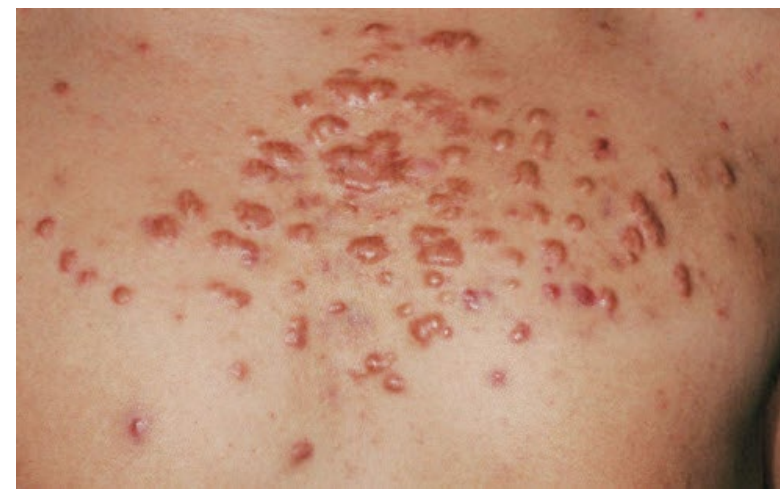

Figure 6. Keloidal scar

\section{Procedures for acne scars}

\section{Noninvasive}

Topical therapy: Topical hypo pigmenting agents like $4 \%$ hydroqinone, $10 \%$ glycolic acid, $20 \%$ azelaic acid, $2 \%$ kojic acid are useful mainly for macular and hyper pigmented scars.

Non-ablative lasers/radiofrequency and light therapy: Ablative and non-ablative fractional lasers may be effective for all types of atrophic acne scars except for deep icepick scars. Often a combination of techniques (e.g. Subcision or filler injections combined with fractional resurfacing) will yield a superior result compared to one procedure alone)

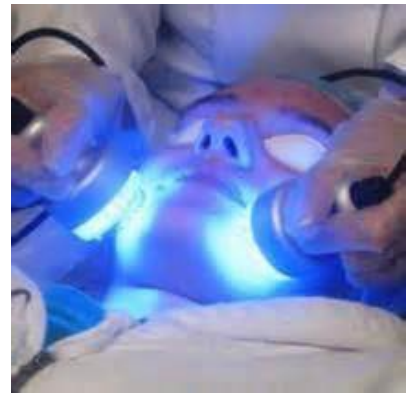

Silicone gel and sheets: Silicone dressings available as gels or sheets are chemically and biologically inert substance is found to be helpful in flattening hypertrophic and keloid acne scars.

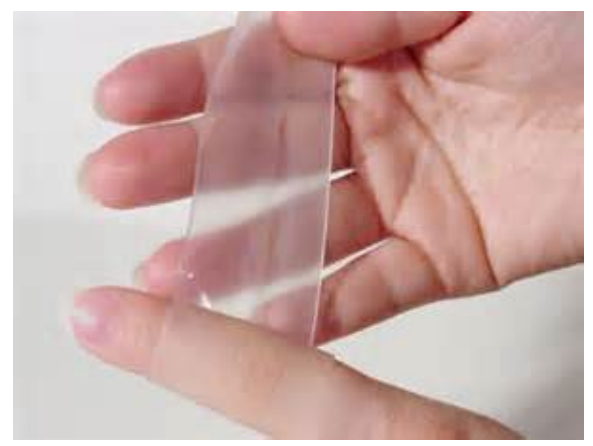

\section{Minimally invasive}

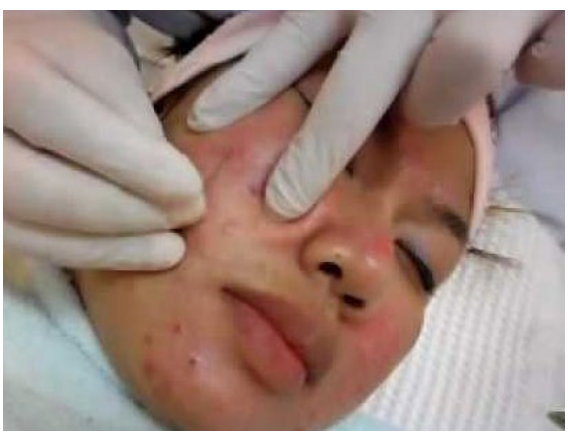

Subcision: Subcision is done for depressed, icepick and rolling scars. The principle of this procedure is to break the fibrotic strands, which tether the scar to the underlying subcutaneous tissue (Figure 7).

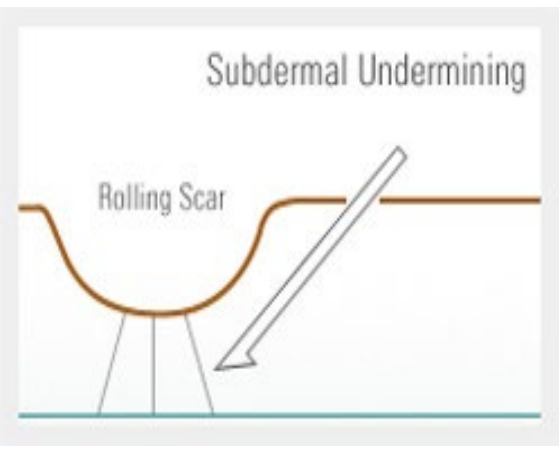

Figure 7. Subcision technique

Chemical peeling: Chemical peeling is a process of causing controlled destruction of the superficial layers of the skin to the required depth, by the application of suitable chemical agent, followed by subsequent remodeling and replacement by normal tissue. The most commonly used chemical peels in treating acne scars include Salicylic Acid (SA), Glycolic Acid (GA) and Trichloroacetic Acid (TCA).

TCA CROSS technique: The CROSS technique is used for ice-pick and narrow boxcar scars. A high-strength trichloroacetic acid (TCA) solution is placed in the base of these scars to ablate the epithelial wall and to promote dermal remodeling (Figure 8). 


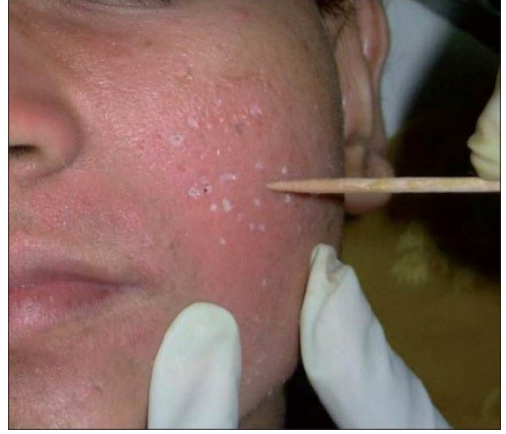

Figure 8. TCA CROSS technique

Microdermabrasion: While not a leading treatment for acne scars, microdermabrasion can be effective for very mild acne scarring. During this procedure, a dermatologist uses a machine to remove the top surface layer of skin. With a series of microdermabrasion sessions, the skin can repair itself and shallow scars tend to become less noticeable.

Skin needling: Skin needling, also called "collagen induction therapy" or "needle dermabrasion" is the technique of rolling a device composed of a barrel studded with hundreds of needles, which create thousands of micropunctures in the skin to the level of the papillary to mid-dermis. The optimal scars to treat with skin lesion are the same as fractional laser resurfacing_rolling acne scars, superficial boxcar scars, or erythematous or hypopigmented macular scars (Figure 9).

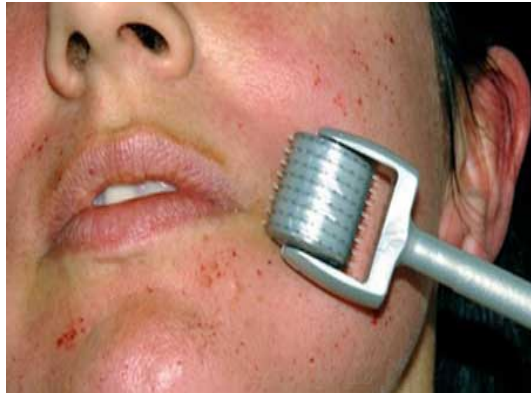

Figure 9. Skin needling

Fillers: Fillers add volume to the skin and are effective in treating patients with depressed soft rolling acne scars. They are not useful for icepick scars. They can be used alone or in combination with other procedures (Figure 10).

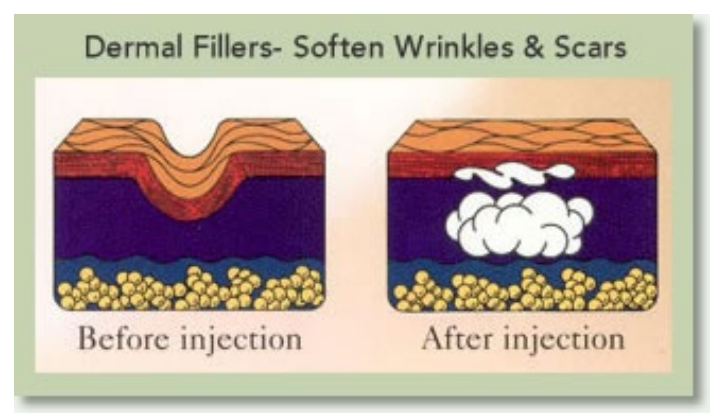

Figure 10. Fillers technique

Intralesional steroids: Individual nodular or cystic acne lesions often dramatically decrease in size after intralesional injection of a corticosteroid. Patients with severe acne often require repeated injections every 2 to 3 weeks. The steroid preparation triamcinolone acetonide $10 \mathrm{mg} / \mathrm{mL}$ (e.g., Kenalog-10) is a preferred agent and should be diluted to about $2.5 \mathrm{mg} / \mathrm{mL}$ with saline or local anesthetic (e.g., $1 \%$ lidocaine) (Figure 11).

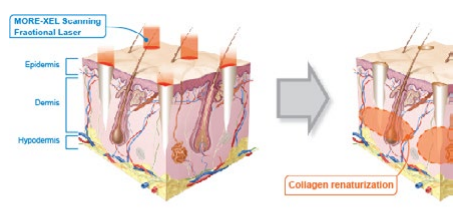

Figure 11. Intralesional steroids technique

Fractional photothermolysis: The treatment delivers a series of microscopic laser spots, simultaneously preserving areas of unaffected, normal skin between. The healthy cells in the untouched spots accelerate rapid healing of the whole area, resulting in faster recovery time and less side effects (Figure 12).
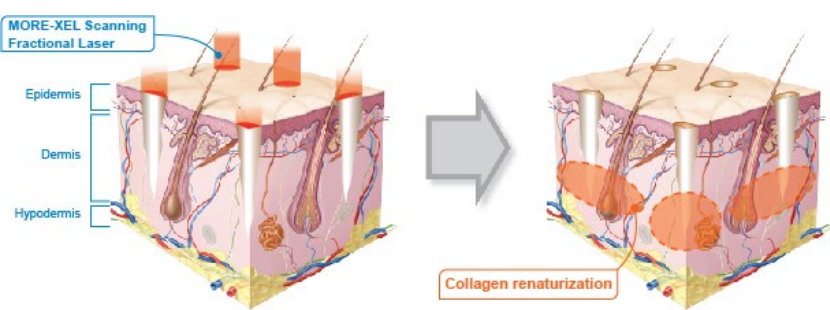

Figure 12. Fractional photothermolysis

InvasivePunch excision technique: The punch excision of icepick acne scars or deep boxcar scars is a technique that can be easily adopted into a dermatology practice. Most dermatologists are often performing punch biopsies for diagnostic purposes of inflammatory dermatoses. The same technique is used to remove appropriate acne scars. A disposable punch biopsy

Instrument is selected that matches the size of the icepick or narrow boxcar scar, including the walls of the scar (Figure 13).

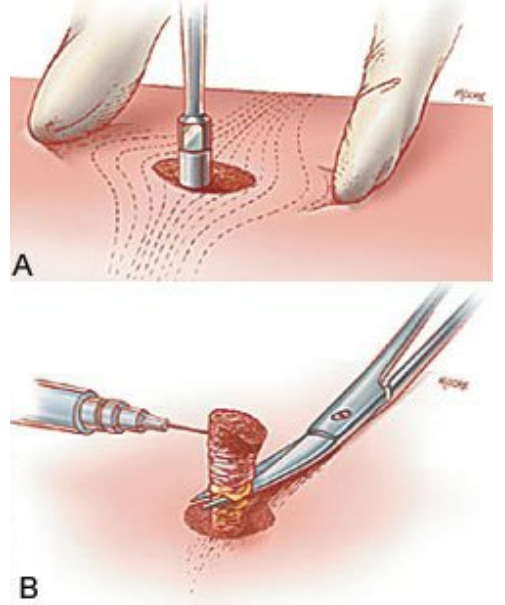

Figure 13. Punch excision technique 
Laser resurfacing: Laser resurfacing is a treatment to reduce facial wrinkles and skin irregularities, such as blemishes or acne scars.
The technique directs short, concentrated pulsating beams of light at irregular skin, precisely removing skin layer by layer. This popular procedure is also called lasabrasion, laser peel, or laser vaporization.

Copyright: $(2015$ Kannangara AP. This is an open-access article distributed under the terms of the Creative Commons Attribution License, which permits unrestricted use, distribution, and reproduction in any medium, provided the original author and source are credited. 